\title{
Nighttime physician staffing improves patient outcomes: yes
}

\author{
David J. Wallace \\ (C) 2016 Springer-Verlag Berlin Heidelberg and ESICM
}

Nighttime physician staffing improves patient outcomes. This is supported by the literature [1-5] and we have all seen it personally. The question is really not whether nighttime intensivists improve outcomes, but rather when, where, how and at what cost. The question hinges on context.

There is abundant evidence that nighttime physician staffing is responsible for improved patient outcomes both directly and indirectly in a variety of settings. Nighttime physician staffing is associated with improved intensive care unit mortality $[1,3,5]$, improved quality of death and dying [2], and alignment of care to patient preferences outside the intensive care unit [4]. Nighttime intensivists improve outcomes for patients with unexpected deterioration after hours, albeit in ways that are difficult to capture in clinical research. Nighttime intensivist staffing also indirectly improves patient care by mitigating the negative effects of cognitive fatigue and sleep deprivation in a daytime-only staffing model [6]. Both cognitive fatigue and sleep deprivation are associated with impaired performance, decreased use of decisional support, compromised patient safety, and physician burnout [7-9]. Nighttime intensivist staffing mitigates some of these effects by allowing the daytime attending to rest at night [6], thereby improving the care that is delivered during the day. Therefore, in the right setting, through both direct impacts on patient care overnight and indirectly by facilitating high quality care during the day, nighttime staffing improves patient outcomes.

Context is equally important for reconciling the results of studies that did not find benefits from nighttime physician staffing. A recent randomized trial of nighttime staffing in

\footnotetext{
*Correspondence: wallacedj@upmc.edu

Department of Critical Care Medicine, University of Pittsburgh School of Medicine, Pittsburgh, USA

For contrasting viewpoints, please go to doi:10.1007/s00134-016-4367-7 and doi:10.1007/s00134-016-4422-4.
}

a large academic medical intensive care unit did not show improved patient survival or length of stay [10]. It is certainly possible (if not likely) that the study's 1-week cycling of daytime-only staffing was not sufficient to unmask the deleterious effects of cognitive and physical fatigue, contributing to a null result. Similarly, 1-week daytime-only staffing cycles would be unlikely to induce physician burnout or emotional exhaustion-domains related to patient safety in critical care that are improved by nighttime staffing $[11,12]$.

A closer look at the University of Pennsylvania study demonstrates how, taken out of the context of avoiding physician burnout or alleviating cumulative cognitive and physical fatigue, the study biased the effect of nighttime staffing towards the null. Figure 1 shows that when an effect of accumulating fatigue is included, there are now four exposure categories. In the study's main analysis, patients $\mathrm{A}$ and $\mathrm{D}$ were considered equivalent: both were admitted to and analyzed in the intervention arm. Similarly, patients $B$ and $C$ were included as controls. However, as the figure shows, patients $\mathrm{A}-\mathrm{D}$ represent different admission scenarios.

Patient A is admitted on a calendar day with nighttime intensivist staffing, yet the daytime attending is still moderately fresh. In this setting, as it relates to the domain of cumulative cognitive and physical fatigue, we would not expect the nighttime intensivist to have a major impact on daytime care. However, patient D is also admitted to a calendar day with nighttime intensivist staffing, yet here the daytime attending is nearing the end of service, and consequently stands the most to benefit from intensivist staffing at night. A similar situation is present for the controls. Patient B is admitted to a calendar day with only daytime coverage and near the end of service, during the highest risk of cumulative fatigue effects. Patient $C$ is admitted to a calendar day with only daytime coverage, but early in the service cycle, with lower attendant risks from fatigue. Again, in the main analysis, patients A and

\section{Springer}




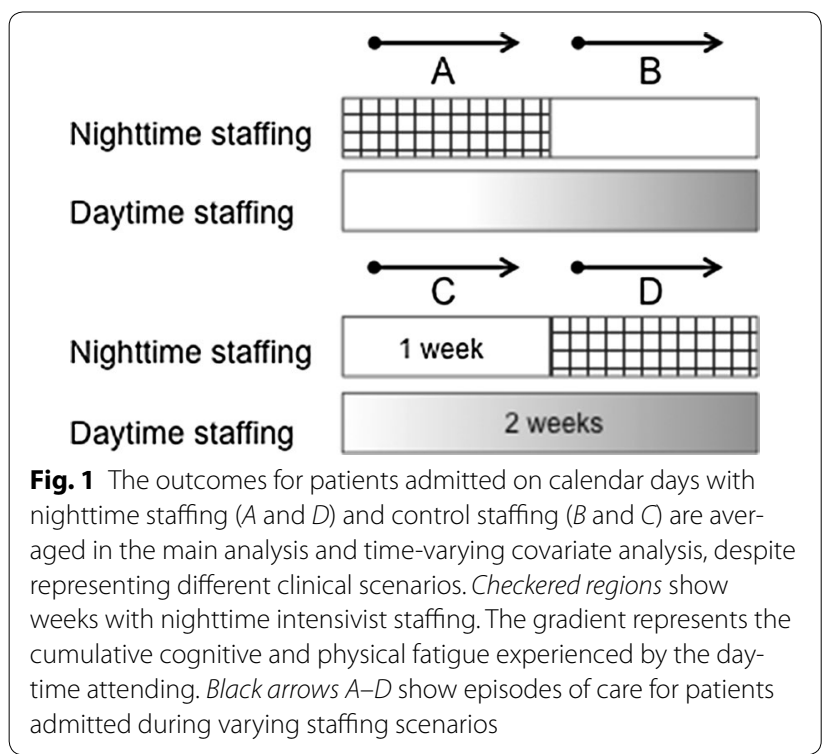

$\mathrm{D}$ are equivalent, as are patients $\mathrm{B}$ and $\mathrm{C}$-despite the fact that each represents a different context for nighttime staffing benefits. By averaging these effects the null result is not surprising and is analogous to exposure misclassification in epidemiologic research [13].

This is not to say that nighttime staffing will always produce improved patient outcomes, if only viewed through the right lens. Nighttime staffing will clearly not improve quality in all circumstances. Furthermore, hospitals need to weigh the value of adding or maintaining nighttime staffing against competing strategies to improve patient care. Finally, hospitals that are considering de-adoption of nighttime staffing face a special challenge: could a change to daytime-only staffing deliver equivalent care? Unfortunately there are no easy answers to these questions, and certainly no answers that do not consider the local motivations for adopting nighttime coverage, careful process and outcome monitoring, and involvement of key stakeholders. Researchers, administrators, and clinicians alike need to be attentive to context when evaluating the data on nighttime intensivist staffingparticularly when it comes to decisions regarding adoption, de-adoption, or maintenance of this service. Your patient outcomes depend on it.

\section{Acknowledgments}

The author receives grant support from NIH NHLBI-K08-HL122478.

\section{Compliance with ethical standards}

\section{Conflicts of interest}

The author declares no conflicts of interest.

Received: 15 April 2016 Accepted: 25 April 2016

Published online: 27 June 2016

\section{References}

1. Wallace DJ, Angus DC, Barnato AE et al (2012) Nighttime intensivist staffing and mortality among critically ill patients. N Engl J Med 366:20932101. doi:10.1056/NEJMsa1201918

2. Reineck LA, Wallace DJ, Barnato AE, Kahn JM (2013) Nighttime intensivist staffing and the timing of death among ICU decedents: a retrospective cohort study. Crit Care 17:R216. doi:10.1186/cc13033

3. Kajdacsy-Balla Amaral AC, Barros BS, Barros CCPP et al (2014) Nighttime cross-coverage is associated with decreased intensive care unit mortality. A single-center study. Am J Respir Crit Care Med 189:1395-1401. doi:10.1164/rccm.201312-21810C

4. Stelfox HT, Hemmelgarn BR, Bagshaw SM et al (2012) Intensive care unit bed availability and outcomes for hospitalized patients with sudden clinical deterioration. Arch Intern Med 172:467-474. doi:10.1001/ archinternmed.2011.2315

5. Blunt MC, Burchett KR (2000) Out-of-hours consultant cover and case-mix-adjusted mortality in intensive care. Lancet 356:735-736. doi:10.1016/S0140-6736(00)02634-9

6. Garland A, Roberts D, Graff L (2012) Twenty-four-hour intensivist presence: a pilot study of effects on intensive care unit patients, families, doctors, and nurses. Am J Respir Crit Care Med 185:738-743. doi:10.1164/ rccm.201109-17340C

7. Killgore WDS (2010) Effects of sleep deprivation on cognition. Prog Brain Res 185:105-129. doi:10.1016/B978-0-444-53702-7.00007-5

8. Lockley SW, Barger LK, Ayas NT et al (2007) Effects of health care provider work hours and sleep deprivation on safety and performance. Jt Comm J Qual Patient Saf 33:7-18

9. Fraser M, Conduit R, Phillips JG (2013) Effects of sleep deprivation on decisional support utilisation. Ergonomics 56:235-245. doi:10.1080/00140 139.2012.760754

10. Kerlin MP, Small DS, Cooney E et al (2013) A randomized trial of nighttime physician staffing in an intensive care unit. N Engl J Med 368:2201-2209. doi:10.1056/NEJMoa1302854

11. Welp A, Meier LL, Manser T (2016) The interplay between teamwork, clinicians' emotional exhaustion, and clinician-rated patient safety: a longitudinal study. Crit Care 20:1 10. doi:10.1186/s13054-016-1282-9

12. Rothschild JM, Landrigan CP, Cronin JW et al (2005) The critical care safety study: the incidence and nature of adverse events and serious medical errors in intensive care. Crit Care Med 33:1694-1700. doi:10.1097/01. CCM.0000171609.91035.BD

13. Wacholder S, Hartge P, Lubin JH, Dosemeci M (1995) Non-differential misclassification and bias towards the null: a clarification. Occup Environ Med 52:557-558 